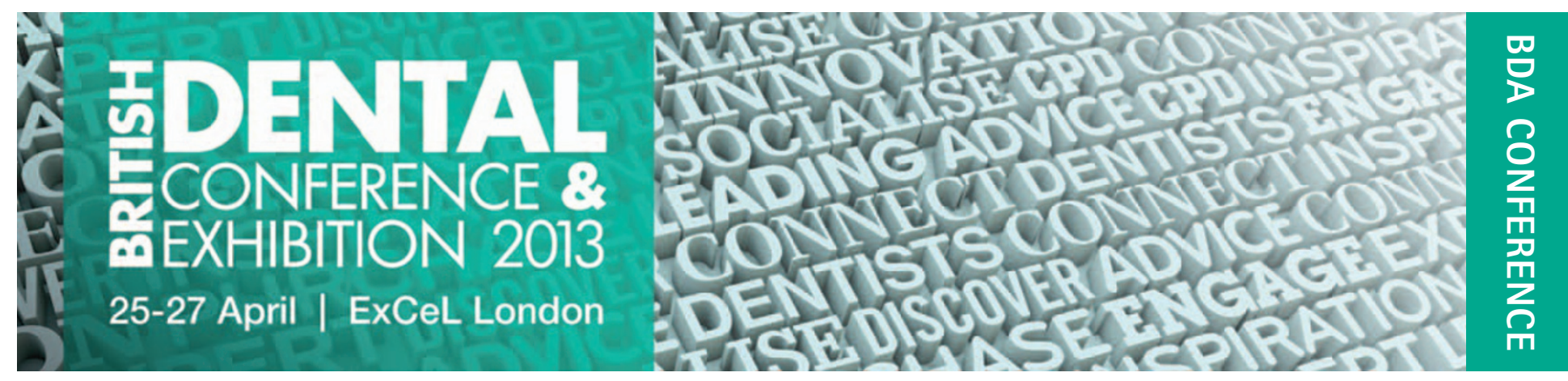

\title{
The 'E' factor - evolving endodontics
}

\author{
M. J. Hunter ${ }^{1}$
}

\begin{abstract}
Endodontics is a constantly developing field, with new instruments, preparation techniques and sealants competing with trusted and traditional approaches to tooth restoration. Thus general dental practitioners must question and understand the significance of these developments before adopting new practices. In view of this, the aim of this article, and the associated presentation at the 2013 British Dental Conference \& Exhibition, is to provide an overview of endodontic methods and constantly evolving best practice. The presentation will review current preparation techniques, comparing rotary versus reciprocation, and question current trends in restoration of the endodontically treated tooth.
\end{abstract}

The challenge in modern-day dental practice is to offer the highest standards of dental care within the budgetary constraints of what the patient can afford whether it is within the NHS or on a private basis. The GDC clearly states that as providers we must put patients' interests first and act to protect them and respect their dignity and choices. ${ }^{1}$ Endodontically, this is a challenging environment and the pressure is on for us to produce a 'perfect canal shape' as quickly as possible, to then fill this space and to restore the tooth. This short article highlights some of the issues that will be addressed at the British Dental Conference and Exhibition next month and aims to encourage the reader to attend for further information.

The ideal root canal preparation tapers from the coronal aspect to the apical constriction while remaining 'centred' and 'transportation' of the canal should be avoided. ${ }^{2}$ It has been possible to achieve such a preparation to this

\footnotetext{
${ }^{1}$ Clinical Teacher in Restorative Dentistry, School of Dentistry, University of Manchester, Manchester, M15 6FH

Correspondence to: Mark Hunter

Email:mark.j.hunter@manchester.ac.uk
}

DOI: 10.1038/sj.bdj.2013.216

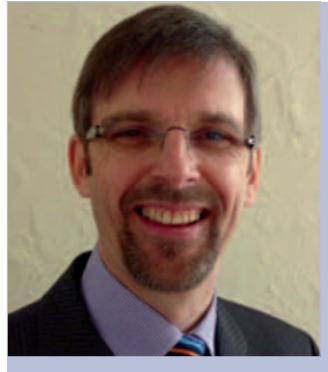

Mark Hunter qualified in 1979 from Manchester University. He divides his time equally between teaching and working in a practice, limited to endodontics, as a GDC registered specialist in South Manchester. His teaching commitment includes one day a week with postgraduates at Manchester University Dental School as a part-time lecturer and clinical teacher, and as a partner in Simplyendo, teaching on programmes affiliated to Chester University to certificate, diploma and degree level in a dedicated endodontic teaching centre in Altrincham, Cheshire. He is also assistant secretary to the British Endodontic Society, with responsibility for prizes and awards.

standard in straight canals with hand files using a 'step back' technique. ${ }^{3}$

The difficulty of managing a curved canal with stainless steel is that as hand files increase in size they tend to remove more tooth tissue on the outer aspect of the curve, which eventually leads to the formation of an 'elbow' and a 'zip'. Such a preparation cannot be filled predictably. The other problem encountered when preparing a curved canal is the tendency to create a blockage, which invariably leads to ledge formation. Before the advent of Ni-Ti instrumentation in the early 1990s, canal preparation in curved canals was conservative and preparation was limited to smaller narrower sizes in an attempt to avoid these complications. Silver cones were used to obturate these finely prepared canals because gutta percha (GP) of a similar dimension would not penetrate to full working length so easily. Silver cone filling 'worked' providing the canal was indeed of a small dimension and also providing the canal was not previously infected. Silver points corrode and associated with poor bacterial control these cases are more likely to fail. ${ }^{4}$

Less preparation, while tending to avoid iatrogenic problems, is not useful in terms of infection control. Historically, to combat problems of infection and lack of thorough canal debridement, sealers that fixed tissues were more common. The toxicity of these agents caused more than a few problems apically if they were placed beyond the confines of the tooth. ${ }^{5}$ Flaring of the canal while maintaining centrality of preparation was the goal to 
enable better infection control and more predictable filling, however, this was a few years before the advent of Ni-Ti and root canal preparation was awaiting an 'evolutionary' step.

Roane, Duncanson and Sabala 'coined' a new term in their paper of 1985, The 'balanced force' concept of instrumentation of curved canals. ${ }^{6}$ Simply stated turning a 'safe-ended' ' $K$ ' file clockwise 90 degrees to 'engage' and then turning between 180 and 360 degrees anticlockwise while maintaining apical pressure allowed the file to 'cut' the canal wall, whether it was straight or curved, without transportation. This was the simple answer to managing curved canals with stainless steel that had been gestating for 13 years before they published their paper.

Why is this an important concept and what is the contemporary relevance? It is the embodiment of a 'forward' and 'reverse' file motion, which is now being adopted in some of the latest $\mathrm{Ni}-\mathrm{Ti}$ preparation techniques where less files are used to produce a shape that hitherto would have required more files to safely produce the same shape.

Can less files do the same work and still create a good canal shape without risking file fracture? It would seem so, if we are to believe the claims made by endodontic instrument manufacturers. During the last two years manufacturers have launched a number of 'new' file systems and exponents of these latest instrument(s) would have us buy into their philosophy (and their new motors!). More established file systems are competing with the 'avantgarde'! We choose to use the more 'tried and tested' conventional Ni-Ti files such as 'RaCe', 'Protaper', 'K3', 'Twisted', 'Revo S', 'BioRaCe', 'MTwo', 'RaCe123', or invest in the next evolutionary step and utilise single file philosophy such as 'Reciproc' and 'WaveOne'. Or we can go 'halfway' and use a single file technique without reciprocation such as 'One Shape'? The list is not exhaustive and yet might we be a little confused, or even overwhelmed?

My intention as an endodontic informer is to review the principles of preparation and to relate the various systems to the creation of the ideal preparation shape and to help guide the "keen to do better' practitioner in making a decision as to which file system suits their

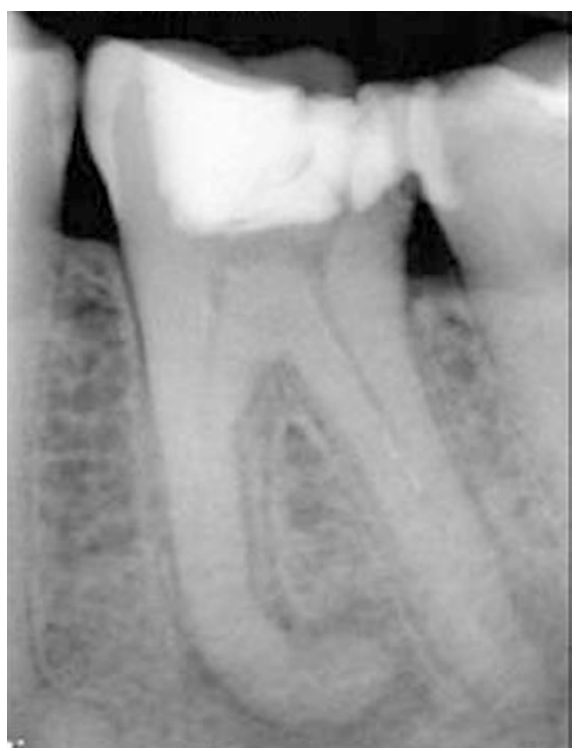

Fig. 1 Pre-operative view LL6 with 'J' shaped mesial root

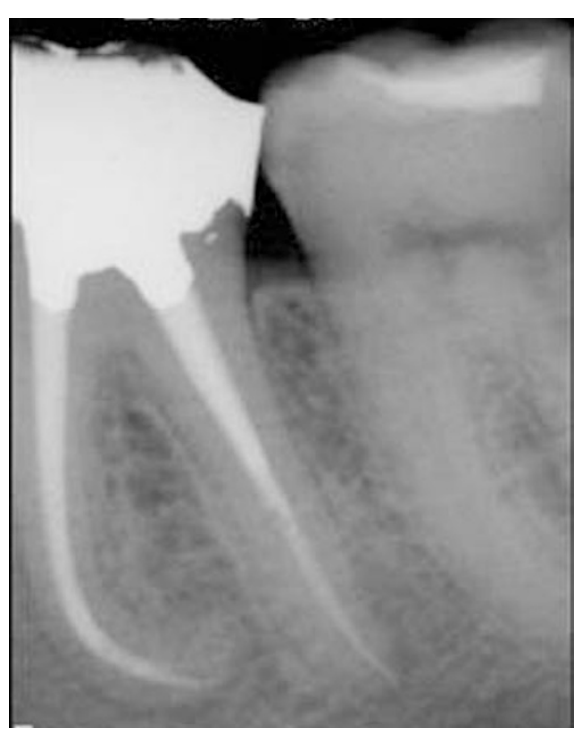

Fig. 2 Completed fill LL6, rotary Ni-Ti files to a FWL of $28 \mathrm{~mm}$ ensured a result that would be much more difficult to prepare using hand files alone

needs. In this context more information is less confusion!

Having created the ideal canal shape, how are we to fill it? Do we obturate after a single visit, or are there factors that determine we complete treatment after a number of visits? We might use 'well-tried, tested and universally taught' cold lateral condensation, which sealer do we choose? Or do we utilise a thermal technique that we are told creates a more homogenous obturation? Or ought we to be using a filling system that creates a single entity within the tooth without different material interfaces reducing the risk of leakage such as 'Resilon' or 'Real Seal' replacing our 'familiar' GP and sealer
- more than a century since its first use?

The apical foramen can be immature, resorbed or just over-prepared. Do we use an 'even more bio-compatible' material such as MTA, Bio-aggregate, BioDentine or MM MTA? How do we place it, what are the pitfalls, the advantages?

If the root canal needs to be re-accessed to retreat the tooth, how do we get to the apex again? Ought we to be embracing the advent of 'bioactive' sealers, which both seal and repair apical foramena, placing a fine single GP cone within the midst of the fill, just in case we need to re-access the canal again? This sounds like a return of the 'single cone technique'. What interesting and changing times!

What teeth do we re-treat, what are the potential outcomes, what do we tell the patient? How realistic are our endodontic abilities in relation to the expectations of our clients? We must decide whether to give the tooth 'a go' or transfer the patient to an endodontic 'wizard' or 'guru' who can get to those parts we cannot reach.

Once the 'Utopian' fill has been placed, we have to consider how to protect the tooth for the rest of the patient's life. We like to think we are that good at endodontics now that the tooth will outlive the patient, but will it? Does our post endodontic restoration support this ideal, or is the choice of restoration likely to influence the outcome positively or adversely and if so, by how much?

Many questions, do we have the answers? Let's see! 'Evolving endodontics' - I look forward to talking you through this 'matrix' of intrigue at the British Dental Conference \& Exhibition. 'The 'E' factor' on Friday, 26 April 16:45! See you there!

1. General Dental Council. Standards for dental professionals. London: GDC, 2005.

2. Schilder $\mathrm{H}$. Cleaning and shaping the root canal. Dent Clin North Am 1974; 18: 269-296.

3. Weine FS, Healey H J, Gerstein H, Evanson L. Pre-curved files and incremental instrumentation for root canal enlargement. J Can Dent Assoc (Tor) 1970; 36: 155-157.

4. Margelos J, Eliades G, Palaghias G. Corrosion pattern of silver points in vivo. J Endod 1991; 17: 282-287.

5. Gluskin A H. Mishaps and serious complications in endodontic obturation. Endodontic Topics 2005; 12: $52-70$.

6. Roane J B, Sabala M, Duncanson M G Jr. The 'balanced force' concept of instrumentation of curved canals. J Endod 1985; 11: 203-211.

Mark Hunter will be speaking on this topic on Friday 26 April at the 2013 British Dental Conference \&t Exhibition held at ExCeL, London. Register at: www.bda.org/conference. 